\title{
Ultra-Widefield Swept-Source Optical Coherence Tomography Findings of Peripheral Retinal Degenerations and Breaks
}

\author{
Ryo Kurobe $\mathbb{D}$ \\ Yoshio Hirano (ID \\ Shuntaro Ogura \\ Tsutomu Yasukawa (iD \\ Yuichiro Ogura \\ Department of Ophthalmology \& Visual \\ Science, Nagoya City University \\ Graduate School of Medical Sciences, \\ Nagoya, Aichi, Japan
}

Correspondence: Yoshio Hirano Department of Ophthalmology \& Visual Science, Nagoya City University Graduate School of Medical Sciences, I-Kawasumi, Mizuho-cho, Mizuho-ku, Nagoya, Aichi, 467-860I, Japan

Tel +8I-52-853825।

Fax +8I-52-84I9490

Email yoshio.hirano@gmail.com
Purpose: To investigate whether ultra-widefield (UWF) swept-source optical coherence tomography (SSOCT) system (Silverstone, Nikon Healthcare Japan, Inc, Tokyo, Japan) can obtain OCT images of peripheral retinal degenerations and breaks.

Methods: Thirty-seven eyes of 31 consecutive cases (16 men, 15 women; mean age 51 years) who had peripheral retinal degenerations and underwent a UWF-SSOCT imaging were enrolled. The convenience and usefulness were investigated.

Results: In all eyes, OCT images were capable and interpretable without any special techniques. The respective findings on OCT were retinal degeneration in 16 eyes: lattice degeneration in 8 eyes, paving stone degeneration in 4 eyes, and unclassified in 4 eyes, retinal tear in 12 eyes, and retinal hole in 9 eyes. The respective locations of the retinal degenerations or breaks were the posterior pole in 0 eyes, mid-periphery in 23 eyes, and far-periphery in 14 eyes. Fifteen eyes had a rhegmatogenous retinal detachment (RRD), one of which was preoperative and the other 14 eyes were postoperative. A buckle was placed in 8 eyes for RRD repair. Subretinal fluid was observed in 9 of 21 eyes with retinal break or retinal hole. Vitreoretinal traction was observed in 10 of 27 eyes without a history of vitrectomy. Inverted artifacts on OCT images were observed in 20 of 37 eyes, which were not related to the location or axial length, but were supposed to be due to the limited scan depth.

Conclusion: The UWF-SSOCT system could capture the OCT images of peripheral retinal degenerations and breaks without any special technique.

Keywords: ultra-widefield, swept-source optical coherence tomography, peripheral retinal degeneration, retinal break, rhegmatogenous retinal detachment

\section{Introduction}

Huang and Fujimoto et al succeeded in drawing a tomographic image of the retina and reported it to Science journal in 1991. ${ }^{1}$ Since the first commercialization of optical coherence tomography (OCT) in 1996, it has played a major role in the evaluation and treatment of retinal diseases centered on the macula. To date, OCT has been widely used not only for retinochoroidal lesions but also for ophthalmic medical care in general, and becomes an indispensable device in ophthalmic medical care.

Many important vitreoretinal abnormalities encountered in vitreoretinal care are located in the retinal periphery, identifiable on skilled peripheral retinal examination using indirect and/or direct fundus ophthalmoscopy, or more recently with the use of 2-dimensional ultra-widefield (UWF) imaging. ${ }^{2,3}$ Previously, the imaging range 
of OCT was limited to the posterior pole of the fundus, but it is gradually expanding. Recently, wide angle sweptsource OCT (SSOCT) (Plex Elite 9000, Carl Zeiss Meditec, Dublin, CA) has been able to assess the midperipheral retinal structure and blood flow in various eye diseases. ${ }^{4,5}$ However, it is still difficult to capture OCT images of the far-peripheral part of the retina. It is well known that there are various artifacts in OCT image scans. ${ }^{6,7}$ Of the OCT artifacts, it has been reported that the peripheral part of the OCT image is partially inverted in some cases, ${ }^{6}$ which is called as a mirror artifact ${ }^{6,7}$ or an inverted artifact. ${ }^{7}$ Mirror artifacts (inverted artifacts) are often detected in myopic eyes or in the peripheral part of nonmyopic eyes due to the scleral curvature, ${ }^{6,7}$ although they only occur from the Fourier transformation used in OCT systems, including spectral-domain and SSOCT., ${ }^{6,7}$

Recently, another device that combines UWF-scanning laser ophthalmoscope (SLO) with a navigated SSOCT (Silverstone, Nikon Healthcare Japan, Inc, Tokyo, Japan) has become commercially available. This device produces an initial high-resolution UWF-SLO fundus image of up to $200^{\circ}$ and uses navigated SSOCT line or volume scans at any location as mapped by UWF-SLO fundus images, which enables to expand the previously limited imaging range to the outermost part of the retina. Since this device can also be photographed with only the basic technology, the spread of this device will make it easier to capture the tomographic images in the retinal periphery, and its evaluation will be useful for clinicians.

In the current study, we investigated whether this device could obtain the OCT images of the peripheral retinal degenerations and/or retinal breaks, and the frequency of inverted artifacts on the OCT images and the relationship with the location of retinal degenerations or axial length were also investigated.

\section{Methods}

\section{Study Design and Setting}

This was a retrospective, observational, consecutive case series conducted in an institutional setting. The study protocol was approved by the Institutional Review Board of Nagoya City University Graduate School of Medical Sciences (No. 60-20-0036). A written informed consent was not obtained from the subjects because of the retrospective nature of this study. Instead, a home page was created with information on the purpose of this study for the subjects to be able to read. The described research methods and analysis adhered to the tenets of the Declaration of Helsinki.

Patients who visited Nagoya City University Hospital from March 2020 through April 2020 were subjected. The inclusion criteria were: eyes with retinal degeneration, retinal break, retinal hole, or retinal detachment. In addition, UWF SSOCT images of retinal peripheral degenerations and/or breaks in a specified area using Silverstone were obtained. The exclusion criteria were patients who do not agree with participation in the current study. The criteria for acquisition of OCT scan images were not set, but patients whose images were too poor quality to be analyzed were excluded. Additionally, cases were also excluded if the specific area with retinal peripheral degenerations were not photographed.

\section{Observational and Assessment Procedure}

All patients underwent a complete ophthalmic examination including measurement of the refractive error (TONOREF ${ }^{\circledR}$ III, Nidek, Gamagori, Japan), the best-corrected visual acuity (BCVA), slit-lamp examination, and indirect ophthalmoscopy. Axial length was measured by IOL Master 500 (Carl Zeiss, Jena, Germany). A recently commercially available Silverstone (Nikon Healthcare Japan, Inc, Tokyo, Japan) was used to acquire the UWF fundus images and OCT images. Silverstone is an ultrawide-field fundus camera equipped with SSOCT, which enables observation of retinal tomography by arbitrary horizontal $23 \mathrm{~mm}$ or $6 \times 3.5 \mathrm{~mm}$ volume scan on the optomap ${ }^{\circledR}$. It was easy to operate the device with only basic training, and no special knowledge or training was required. Since an OCT image at an arbitrary point can be obtained after taking UWF fundus photographs, it is also very useful when examining the image later. The imaging procedure using Silverstone was performed by a clinician ( $R$. $\mathrm{K}$ ) or photographers. The photographers received an instruction prior to the examination by the clinicians about where to be imaged and captured the OCT image of the targeted area using UWF HD volume scan mode $(6 \times 3.5 \mathrm{~mm})$.

The peripheral retinal degenerations were classified by two retinal specialists (R.K. and Y.H.) into lattice degeneration, microcystoid degeneration, paving stone degeneration, retinal hole, and retinal tear according to ICD-10. ${ }^{8}$

The location of retinal peripheral degenerations was determined with UWF fundus photographs, eg, posterior pole was defined as retina within the major temporal vascular arcades and slightly just beyond. ${ }^{9-12}$ Mid-periphery was defined as retina extending from the vascular arcades to the posterior edge of vortex vein ampulla. Far-periphery was 
defined as anterior edge of vortex vein ampulla and beyond to pars plana as previously reported..$^{9-12}$

SSOCT images of peripheral retinal lesions were acquired with this device (Silverstone) and the SSOCT findings around the peripheral retinal degenerations such as the presence of subretinal fluid (SRF) and presence of vitreoretinal traction were investigated.

Furthermore, the frequency of inverted artifacts in the OCT images and the relationship with the location of retinal degenerations or axial length were also investigated.

\section{Statistics}

All values were expressed as the mean \pm standard deviation (SD). BCVA was measured using a Landolt chart and the decimal visual acuity was converted to the logarithm of the minimum angle of resolution ( $\log M A R$ ) units for the statistics. Statistical analyses were performed using Easy R (EZR) software Ver. 1.40 (Jichi Medical University Saitama Medical Center, Saitama, Japan) and $\mathrm{p}<0.05$ was set as significantly different. The $\chi^{2}$ test was used to compare whether the OCT image was inverted for the midperipheral lesions and far-peripheral lesions. An unpaired$t$ test was used to compare the mean axial length between the inverted and the noninverted group. Spearman's rank correlation coefficient was used to evaluate a correlation between the axial length and the presence of inverted OCT image.

\section{Results \\ Patients}

Thirty-eight eyes of 32 patients with peripheral retinal degeneration underwent UWF imaging using Silverstone. One eye did not meet the study criteria: a photographer did not capture the specified area with retinal degeneration but mistakenly imaged a wrong area without any retinal degenerations. Thus, 37 eyes of 31 patients ( 15 men, 16 women; mean age, $51.0 \pm 18.8$ years; range, $16-82$ years) were enrolled. The patient characteristics are shown in Table 1. The mean LogMAR VA was $-0.0277 \pm 0.199$. The mean refractive error was $-4.54 \pm 3.49$ diopters. The axial length was measured in 26 eyes, and the mean value was $25.3 \pm 1.58 \mathrm{~mm}$. The lens status was phakia in 25 eyes and pseudophakia in 12 eyes.

\section{Representative Cases}

The UWF color fundus images and UWF-SSOCT findings of the representative cases are shown in Figures 1, 2, and 3.
Table I Demographics of the Study Population with Peripheral Retinal Degenerations

\begin{tabular}{|l|c|}
\hline Eyes/patients, $\mathrm{n}$ & $37 / 3 \mathrm{I}$ \\
Male & $17 / 16$ \\
Female & $20 / 15$ \\
Mean age (SD), years & $51.0(18.8)$ \\
Range & 16 to 82 \\
Decimal VA (Range) & 0.15 to 1.5 \\
Mean LogMAR VA (SD) & $-0.0277(0.199)$ \\
Mean refractive error (SD), diopters & $-4.54(3.49)$ \\
Range & -11.5 to 0 \\
Mean axial length (SD), mm & $25.3(1.58)$ \\
Range & 22.2 to 27.9 \\
Lens status (phakia/pseudophakia/aphakia) & $25 / 12 / 0$ \\
Type of peripheral retinal degeneration and the & - \\
location, (Mid-periphery/Far-periphery), $\mathrm{n}$ & \\
Degeneration (lattice) & $8(4 / 4)$ \\
Degeneration (paving stone) & $4(3 / 1)$ \\
Degeneration (unclassified) & $4(3 / 1)$ \\
Tear & $12(7 / 5)$ \\
Hole & $9(6 / 3)$ \\
Rhegmatogenous retinal detachment (Y/N), $\mathrm{n}$ & $15 / 22$ \\
Treatment, $\mathrm{n}$ & - \\
None & 10 \\
Laser alone & 11 \\
Buckling & 6 \\
Vitrectomy & 8 \\
Vitrectomy with Buckle & 2 \\
\hline
\end{tabular}

Abbreviations: $\mathrm{N}$, number; SD, standard deviation; VA, visual acuity; LogMAR, logarithm of the minimum angle of resolution; $\mathrm{Y}$, yes; $\mathrm{N}$, no.

\section{Type of Peripheral Retinal Degenerations, the Location, and Treatment}

The type of peripheral retinal degenerations, the location, and treatment are shown in Table 1. Retinal degeneration was observed in 16 eyes: lattice degeneration in 8 eyes, paving stone degeneration in 4 eyes, and the others in 4 eyes could not be classified. Retinal tear was observed in 12 eyes: all 12 eyes had a horseshoe retinal tear. Retinal hole was observed in 9 eyes: 5 eyes had an atrophic hole, 2 eyes had an operculated hole, and 2 eyes had a lattice hole.

Of 16 eyes with retinal degeneration, 10 eyes had retinal degeneration in the mid-periphery and 6 in the farperiphery. Of 12 eyes with retinal tear, 7 eyes had retinal tear in the mid-periphery and 5 eyes in the far-periphery. Of 9 eyes with retinal hole, 6 eyes had retinal hole in the mid-periphery and 3 eyes in the far-periphery.

There were 15 eyes of 37 cases with a rhegmatogenous retinal detachment (RRD), 14 eyes of which were postoperative and 1 eye was preoperative. The treatments for 

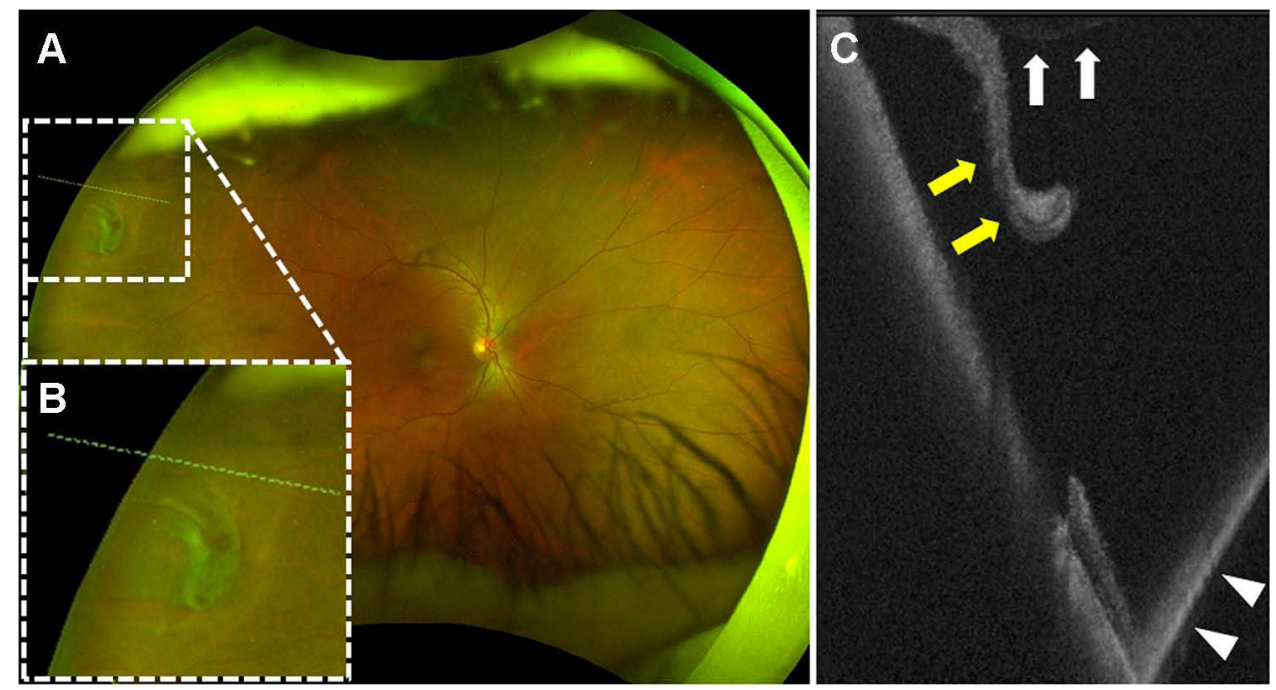

Figure I A multimodal imaging of a 54-year-old male with a peripheral retinal tear in the right eye. (A) An ultra-widefield fundus photograph. A retinal tear is observed in the far-peripheral area (white-dotted square). (B) A magnified image of the yellow-dotted square on (A). (C) An ultra-widefield swept-source optical coherence tomography $(O C T)$ image of the yellow-dotted line (A and $\mathbf{B}$ ). A vitreoretinal traction (white arrows) is observed and a retinal flap is detached from the retina (yellow arrows). Inverted OCT image is also observed (white arrowheads).
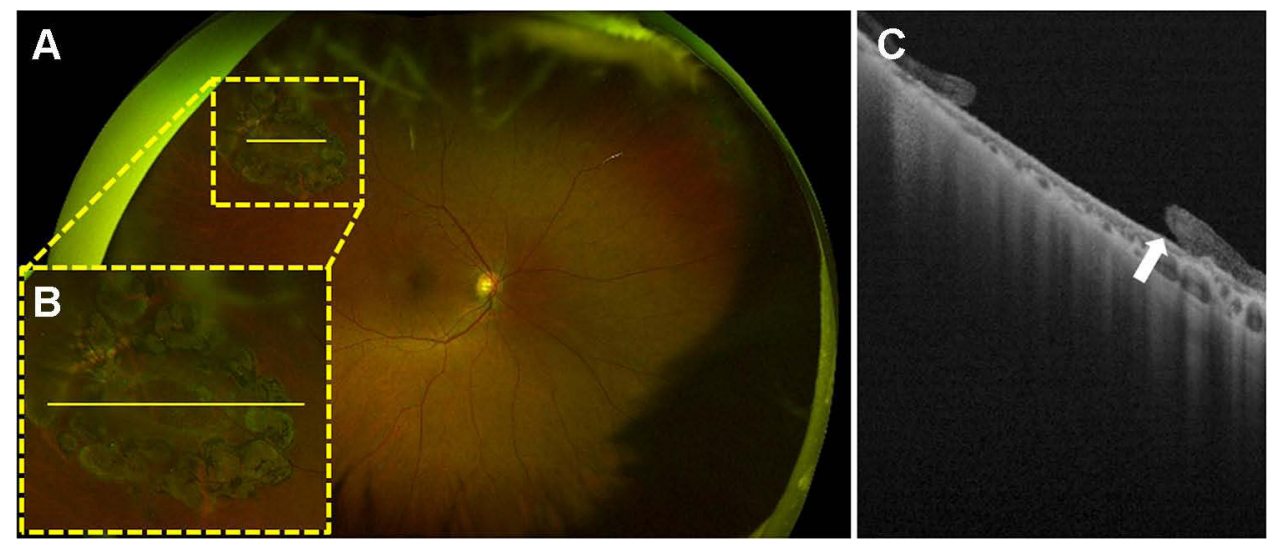

Figure 2 A multimodal imaging of a 74-year-old female with a peripheral retinal hole treated by a laser photocoagulation in the right eye. (A) An ultra-widefield fundus photograph. A retinal hole surrounded by laser scars is observed in the mid-peripheral area (yellow-dotted square). (B) A magnified image of the yellow-dotted square on (A). (C) An ultra-widefield swept-source optical coherence tomography image of the yellow line (A and B). The edge of the retinal hole is slightly detached (white arrow).

the peripheral retinal degenerations were no treatment in 10 eyes, laser photocoagulation in 11 eyes, buckling in 6 eyes, vitrectomy in 8 eyes, and vitrectomy with buckle in 2 eyes. A silicone buckle was placed in 8 eyes.

\section{Ultra-Widefield Swept-Source Optical Coherence Tomography Findings at the Peripheral Retinal Degenerations}

The UWF-SSOCT findings are shown in Table 2. SRF was observed in 9 eyes of 21 eyes with retinal tear or retinal hole, 4 eyes of which had a retinal tear and 5 had a retinal hole. A vitreoretinal traction was observed in 10 eyes of 27 eyes without a history of vitrectomy, 4 eyes of which had a retinal degeneration, 3 eyes had a retinal tear, and 3 eyes had a retinal hole.

\section{Inverted Artifacts in Optical Coherence Tomography Images}

The representative inverted artifacts in the UWF-SSOCT images are shown in Figure 4. In the current study, the inverted artifacts were observed in 20 eyes of 37 eyes. The inverted artifacts were found in 10 eyes of 23 eyes with mid-peripheral lesions and 10 eyes of 14 eyes with farperipheral lesions. There was no significant difference $(\mathrm{P}=$ 0.173 ). In addition, there was no significant difference in the mean axial length between the inverted group and the 

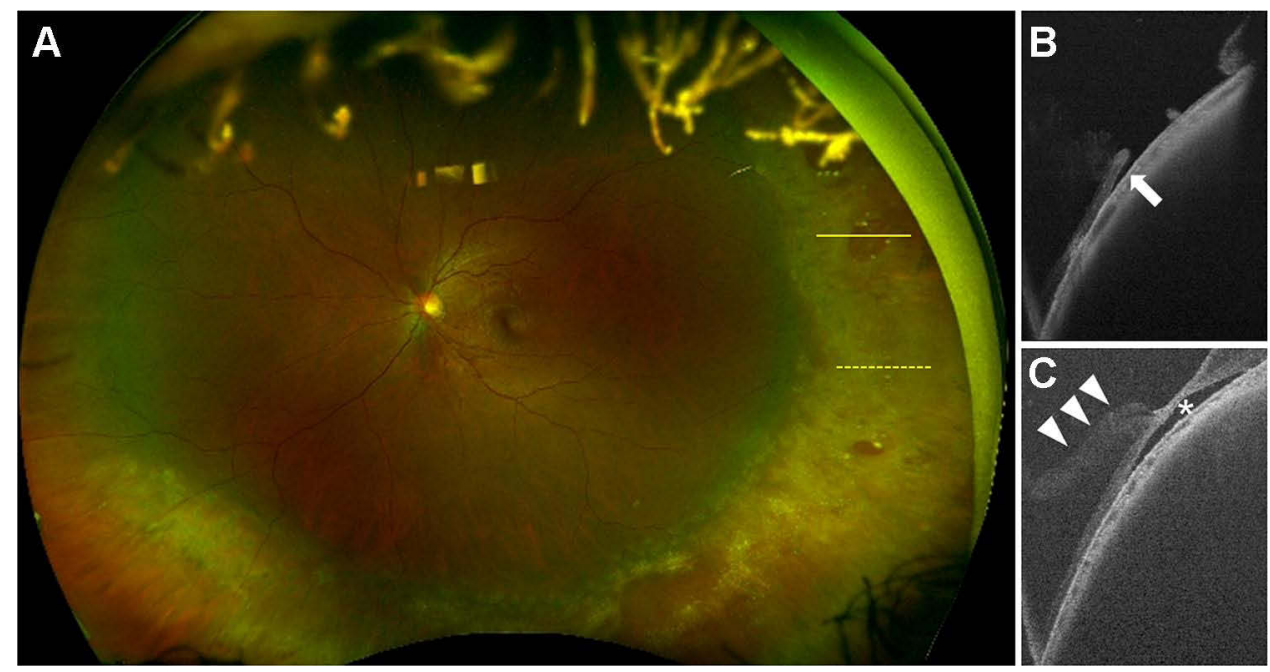

Figure 3 (A) A multimodal imaging of a 26-year-old female with a rhegmatogenous retinal detachment treated with an encircling buckling in the left eye. (A) An ultrawidefield fundus photograph. A protrusion of the encircling buckle is observed. Retinal holes (yellow arrows) are observed on the protrusion of the buckle. (B) An ultrawidefield swept-source optical coherence tomography (UWF-SSOCT) image of the yellow line on (A). The edge of the retinal hole is slightly detached (white arrow). (C) An UWF-SSOCT image of the yellow-dotted line on (A). A vitreoretinal traction (white arrowheads) is observed and a remnant subretinal fluid (white asterisk) is also observed. A gentle curve due to the buckle is recognized on (B and $\mathbf{C}$ ).

noninverted group $(\mathrm{P}=0.921)$. Furthermore, no correlation was found between the axial length and whether the OCT image was inverted or not $(\mathrm{r}=0.0462, \mathrm{P}=0.823)$.

\section{Discussion}

OCT provides us with high-definition images as if they were pathological photographs. It is very useful in clinical practice and sometimes plays an important role in decision-making. Specifically, clinicians frequently encounter a membranous structure on the retina on an OCT image despite the presence of an apparent posterior vitreous detachment with Weiss ring in eyes with high myopia. ${ }^{13}$ Also, the differentiation of retinoschisis from retinal detachment can be difficult to understand with an indirect ophthalmoscope alone. ${ }^{2}$ In such cases, OCT images provide very useful information. ${ }^{2}$ The problem of OCT imaging is that the imaging area was localized to the posterior pole of the fundus, but the field of view is expanding as the technology improves.

Table 2 The Ultra-Widefield Swept-Source Optical Coherence Tomography Findings Around the Peripheral Retinal Degenerations

\begin{tabular}{|l|c|}
\hline Subretinal fluid (Y/N), n & $9 / 28$ \\
Edge detached (Y/N/NA), $n$ & $9 / 12 / 16$ \\
Vitreous traction (Y/N/NA), n & $10 / 17 / 10$ \\
Inverted image (Y/N), n & $20 / 17$ \\
\hline
\end{tabular}

Abbreviations: Y, yes; N, no; NA, not applicable.
In the current study, we investigated whether a UWFSSOCT can capture the OCT images of peripheral retinal degenerations and breaks.

Regarding the UWF-SSOCT imaging, anyone could perform without any special technologies as long as the lesion and imaging mode were specified in the photographer. This is very beneficial because clinicians do not have to perform the imaging by themselves or observe the procedure during the imaging. It was possible to photograph and evaluate the OCT images in all cases without any problems, except for one case in which the photographer imaged a wrong area without any retinal degenerations, which was just a mistake and not the technical issue.

In the current study, we found that the lesions of peripheral retinal degenerations were mid-periphery in 23 eyes and far-periphery in 14 eyes. SRF was observed in 9 eyes, of which 4 eyes had a retinal tear and 5 eyes had a retinal hole. Vitreoretinal traction was observed in 10 of 27 eyes that had not undergone a vitrectomy surgery: 3 eyes had a retinal degeneration, 4 eyes had a retinal tear, and 3 eyes had a retinal hole. Regarding the visualization of vitreous, it was not as well as we expected although the OCT system is a swept-source style. It is unclear whether it is because the vitreous was really detached from the retina or there are some functional problems with the UWF-SSOCT.

Inverted artifacts in OCT images were observed in 20 eyes of 37 eyes (54\%). There were no particular problems with a decision-making or image judgement, but it is 


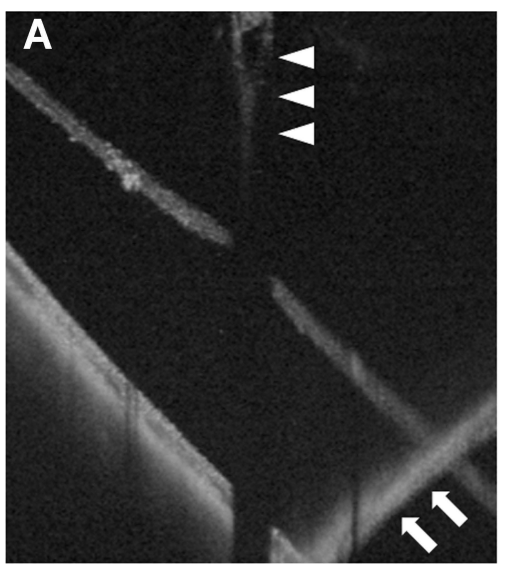

B

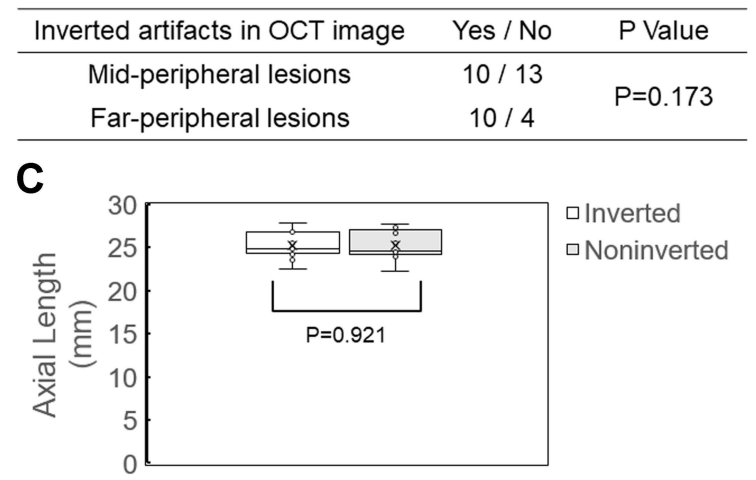

Figure 4 An inverted optical coherence tomography image. (A) Swept-source optical coherence tomography (OCT) image of the peripheral retinal tear. A vitreoretinal traction is observed (white arrowheads). The OCT image is inverted (white arrows). (B) Inverted artifacts in OCT image related to the location of the retinal degenerations. There was no significant difference between the mid-periphery and far-periphery $(P=0.173)$. (C) The mean axial length of the inverted and noninverted group. There was no significant difference $(\mathrm{P}=0.921)$.

a problem that should be solved. Neither the location of the retinal degenerations nor the axial length was correlated with the inversion of OCT images. The inverted artifacts only occur from the Fourier transformation used in OCT systems with Fourier-domain detection, ${ }^{7}$ which is a problem to be solved. The scan depth of the UWFSSOCT (Silverstone) is as short as $2.5 \mathrm{~mm}$. Therefore, it is presumed to be due to the fact that the scan depth may exceed the limit at the peripheral part due to the structure of the eyeball. Another device with a scanning depth of $5 \mathrm{~mm}$ is also on the market, and it seems to be necessary to compare them.

While the overall incidence of atrophic hole and lattice-associated retinal detachment is low, these lesions are present in a disproportionate number of patients with RRD. ${ }^{2,14-16}$ Although it was not clarified in the current study, it would be great if we can know what kind of lattice degeneration, retinal tear, or retinal hole is at the high risk of developing RRD and what kind of findings are active and what kind of findings are silent with the OCT findings, which may provide useful information when a prophylactic laser for these lesions should be applied.

In the future, it will be useful to observe the longitudinal OCT findings until the onset of RRD and the absorption process of residual SRF after RRD repair surgery.

The current study had several limitations. The current study was a retrospective design with a small number of cases. There is no comparison between cases with high myopia and cases without high myopia, or between cases with degenerative lesions and cases without degenerative lesions.

In conclusion, we could capture the OCT images of peripheral retinal degenerations and breaks using UWFSSOCT. However, the OCT findings of peripheral retinal degenerations and breaks are still unclear, and it is necessary to examine them with a prospective, randomized, controlled study in a large number of cases. Also, new findings in a variety of other diseases may bring fundamental changes in treatment and diagnosis.

In addition, the current study performed only one-time evaluation of the OCT images, and the longitudinal changes may provide us with new findings. If there is a difference in the risk of progression of RRD depending on the presence or degree of vitreous traction to the degenerated area and tiny SRF around the retinal break, it may lead to new therapeutic criteria, such as prophylactic retinal laser photocoagulation. UWF-SSOCT may bring advances in the treatment of retinal disorders through ongoing research.

\section{Acknowledgments}

The 59th Annual Meeting of Japanese Retina and Vitreous Society, Fukuoka, Japan, November 27-29th, 2020. We thank Fumie Shibuya, Sayaka Oshio, Satomi Izumi, and Miho Takeuchi (Nagoya City University Graduate School of Medical Sciences) for contribution to data collection.

\section{Funding}

There is no funding to report. 


\section{Disclosure}

Y.H. was supported by a Grant-in-Aid for Scientific Research (C) 18K09416 and 21K09678 from the Japan Society for the Promotion of Science, Tokyo, Japan and the Hori Sciences \& Arts Foundation, Nagoya, Japan. The Japan Society for the Promotion of Science also supported S.O. [Grant-in-Aid for Research Activity Start-up 20K22785], T.Y. [Grant-in-Aid for Scientific Research (C) 19K09954]. Y.O. reports grants and personal fees from Novartis; personal fees from Bayer, Senju Pharma., Chengdu Kanghong Biotechnology, Kyoto Drug Discovery \& Development, Chugai Pharmaceutical Co., Iveric Bio, Apellis Pharmaceutical Co., Topcon, and Carl Zeiss; grants from Boehringer Ingelheim, outside the submitted work. The authors report no other conflicts of interest in this work.

\section{References}

1. Huang D, Swanson EA, Lin CP, et al. Optical coherence tomography. Science. 1991;254:1178-1181. doi:10.1126/science.1957169

2. Kovacs KD, Mahrous MA, Gonzalez L, et al. Feasibility and clinical utility of ultra-widefield-navigated swept-source optical coherence tomography imaging. J Vitreoretin Dis. 2021:2474126421997335. doi:10.1177/2474126421997335

3. Fogliato G, Borrelli E, Iuliano L, et al. Comparison between ultra-widefield pseudocolor imaging and indirect ophthalmoscopy in the detection of peripheral retinal lesions. Ophthalmic Surg Lasers Imaging Retina. 2019;50:544-549. doi:10.3928/23258160-20190905-02

4. Miyata M, Oishi A, Hasegawa T, et al. Concentric choriocapillaris flow deficits in retinitis pigmentosa detected using wide-angle swept-source optical coherence tomography angiography. Invest Ophthalmol Vis Sci. 2019;60:1044-1049. doi:10.1167/iovs.18-26176

5. Glacet-Bernard A, Miere A, Houmane B, et al. Nonperfusion assessment in retinal vein occlusion: comparison between ultra-widefield fluorescein angiography and widefield optical coherence tomography angiography. Retina. 2021;41:1202-1209. doi:10.1097/IAE.00 00000000002993
6. Ho J, Castro DP, Castro LC, et al. Clinical assessment of mirror artifacts in spectral-domain optical coherence tomography. Invest Ophthalmol Vis Sci. 2010;51:3714-3720. doi:10.1167/iovs.09-4057

7. Bazvand F, Ghassemi F. Artifacts in macular optical coherence tomography. J Curr Ophthalmol. 2020;32:123-131. doi:10.4103/ JOCO.JOCO 8320

8. ICD-10 Version: 2019 [homepage on the Internet]. Chapter VII Disease of the eye and adnexa (H00-H59). Available from: https:// icd.who.int/browse10/2019/en\#/H35.0. Accessed December 8, 2021.

9. Choudhry N, Duker JS, Freund KB, et al. Classification and guidelines for widefield imaging: recommendations from the International Widefield Imaging Study Group. Ophthalmol Retina. 2019;3:843-849. doi:10.1016/j.oret.2019.05.007

10. Patel SN, Shi A, Wibbelsman TD, et al. Ultra-widefield retinal imaging: an update on recent advances. Ther Adv Ophthalmol. 2020;12:1-12.

11. Yasuda Y, Hirano Y, Esaki Y, et al. Peripheral microvascular abnormalities detected by wide-field fluorescein angiography in eyes with branch retinal vein occlusion. Ophthalmic Res. 2019;61:107-114. doi:10.1159/000488496

12. Kurobe R, Hirano Y, Niwa N, et al. Wide-field fluorescein and indocyanine green angiography findings in the eyes with Vogt-Koyanagi-Harada disease. $J$ Ophthalmic Inflamm Infect. 2017;7:16. doi:10.1186/s12348-017-0134-3

13. Takahashi H, Tanaka N, Shinohara K, et al. Ultra-widefield optical coherence tomography imaging of posterior vitreous in eyes with high myopia. Am J Ophthalmol. 2019;206:102-112. doi:10.1016/j. ajo.2019.03.011

14. Byer NE. Long-term natural history of lattice degeneration of the retina. Ophthalmology. 1989;96:1396-1401. doi:10.1016/S01616420(89)32713-8

15. Winslow RL, Tasman W. Juvenile rhegmatogenous retinal detachment. Ophthalmology. 1978;85:607-618. doi:10.1016/S01616420(78)35641-4

16. Folk JC, Arrindell EL, Klugman MR. The fellow eye of patients with phakic lattice retinal detachment. Ophthalmology. 1989;96:72-79. doi:10.1016/S0161-6420(89)32926-5
Clinical Ophthalmology

\section{Publish your work in this journal}

Clinical Ophthalmology is an international, peer-reviewed journal covering all subspecialties within ophthalmology. Key topics include: Optometry; Visual science; Pharmacology and drug therapy in eye diseases; Basic Sciences; Primary and Secondary eye care; Patient Safety and Quality of Care Improvements. This journal is indexed on PubMed

\section{Dovepress}

Central and CAS, and is the official journal of The Society of Clinical Ophthalmology (SCO). The manuscript management system is completely online and includes a very quick and fair peer-review system, which is all easy to use. Visit http://www.dovepress.com/ testimonials.php to read real quotes from published authors. 